\title{
Vorrang für zivile Friedensförderung - Empfehlungen
}

\author{
Cornelia Brinkmann*
}

\begin{abstract}
After 10 years, the Action Plan "Civilian Crisis Prevention, Conflict Resolution and Post-Conflict Peace-Building" is still a worldwide unique instrument. However, it is characterized by obstacles: There is no unifying guiding principle for peace promotion, and it lacks public visibility and concrete implementation into practice. In reality, the civilian component of promoting peace is often subordinate to the military component. Civilian crisis prevention should thus have priority and be strengthened. To achieve this, conceptual approaches should be developed further, local peace potentials should be strengthened and all interventions should be conflict-sensitive and value-oriented.
\end{abstract}

Keywords: Aktionsplan, Zivile Konfliktbearbeitung, Peacebuilding, konfliktsensibel

Stichworte: Action plan, civilian crisis management, peacebuilding, conflict-sensitive

\section{Einführung}

Die Bundesregierung hat am 12. Mai 2004 den ressortübergreifenden Aktionsplan „Zivile Krisenprävention, Konfliktlösung und Friedenskonsolidierung" verabschiedet. Ziel des Aktionsplans ist es, Krisenprävention als politische Querschnittsaufgabe auf staatlicher und gesellschaftlicher Ebene zu etablieren. Gleichzeitig wurden Wege aufgezeigt, Institutionen und Instrumente der Krisenprävention zu schaffen oder auszubauen und kohärenter als bisher einzusetzen. Der Aktionsplan erkennt die besondere Rolle zivilgesellschaftlicher Akteure in der Krisenprävention an, daher sind im Beirat zivilgesellschaftliche Organisationen vertreten (u.a. Plattform Zivile Konfliktbearbeitung, Berghof Foundation, Deutsches Institut für Entwicklungspolitik, Gemeinsame Konferenz Kirche und Entwicklung, Hessische Stiftung Friedens- und Konfliktforschung, Bonn International Center for Conversion).

Der Aktionsplan ist auch nach zehn Jahren ein weltweit einmaliges Instrument. Den Zivilen Friedensdienst, gefördert aus BMZ-Mitteln, gibt es ebenfalls nur in Deutschland. Das Sekundierungsgesetz und der Tag des Peacekeepers mit Ehrung von zivilem Personal sind in den letzten Jahren neu hinzugekommen, um ziviles Personal in internationalen Missionen besser abzusichern und seine Verdienste sichtbar zu machen. Mit AA-Mitteln werden aus dem Förderprogramm zivik des Instituts für Auslandsbeziehungen ebenfalls zivile Projekte gefördert. In den letzten Jahren etablierten sich darüber hinaus Studiengänge mit Qualifizierungsangeboten der Zivilen Konfliktbearbeitung. Das BMZ fordert zudem in seinem Strategiepapier „Entwicklung für Frieden und Sicherheit“ (2013) von seinen Durchführungsorganisationen (Gesellschaft für Internationale Zusammenarbeit, Kreditanstalt für Wiederaufbau) für alle Projekte, die in Konfliktkontexten durchgeführt werden, ein verpflichtendes Peace and Conflict Assessment (PCA). Diese Analyse von Projekten und ihres Kontexts sowie ihres Wirkens auf Konfliktlinien ist ein essentieller Schritt, um nicht unbeabsichtigt zur Eskalation von Konflikten beizutragen und um vorhandene Friedenspotenziale zu fördern.

Trotz dieser Errungenschaften ist der Aktionsplan von gewissen Dilemmata geprägt:

- Der Aktionsplan ist sehr ambitioniert und formuliert sehr hohe Ansprüche, jedoch fehlen angemessene Ressourcen um diese Ansprüche zu realisieren. Ein verbindendes Leitbild gemeinsamer ziviler Friedensförderung steht aus, um die Zusammenhänge von Konzepten, Akteuren, Verantwortlichkeiten und Aktivitäten zu beschreiben.

- Der Aktionsplan und seine Organe leiden an fehlender Sichtbarkeit. So gibt es z.B. keine genaueren öffentlich verfügbaren Informationen zur Zusammensetzung und Arbeit des Beirats.

- Teilweise sind die Informationen und Konzepte des Aktionsplans schwer verständlich und sehr theorielastig. Es fehlt eine konkrete Übersetzung für die Praxis.

- Die Umsetzungsberichte der Bundesregierung lassen eine Fehlerkultur vermissen, die aber notwendig ist, um aus Vergangenem lernen zu können.

\section{Empfehlungen vom Unterausschuss ZKP, Beirat und Organisationen im Beirat}

Folgende Dokumente mit Empfehlungen zur Weiterentwicklung des Aktionsplans liegen bereits vor:

- Abschlussdokument des Unterausschusses „Zivile Krisenprävention und vernetzte Sicherheit" der 17. Wahlperiode,

- Memorandum des Beirats Zivile Krisenprävention zur Bundestagswahl 2013,

- Plattform Zivile Konfliktbearbeitung - Stellungnahme zum 3. Umsetzungsbericht,

- Plattform Zivile Konfliktbearbeitung - Zivile Krisenprävention - Parlamentarische Aufgabe und Verantwortung (2012),

- GKKE - Kommentar zum 3. Bericht der Bundesregierung zur Umsetzung des Aktionsplans.

Darüber hinaus werden im Folgenden Lehren aus der Praxis der Friedensförderung erläutert und deren Bedeutung für die konkrete Weiterentwicklung des Aktionsplans skizziert.

\section{Lehren aus der Praxis der Friedensförderung}

Trotz der Betonung der zivilen Komponente im Aktionsplan und den Fortschritten der letzten zehn Jahre, wird in der Re-

\footnotetext{
Cornelia Brinkmann, M.A., Erziehungswissenschaftlerin, Kauffrau und Organisationsentwicklerin, ist Geschäftsführerin von Steps for Peace - Institut für Peacebuilding in Berlin, www.steps-for-peace.org.
} 
alität die zivile Komponente oftmals als nachgeordnet zur militärischen dargestellt. Die besondere Bedeutung der zivilen Komponente der Krisenprävention und Konfliktbearbeitung sollte jedoch Vorrang haben und weiter ausgebaut werden. Aus der praktischen Arbeit mit zivilgesellschaftlichen und staatlichen Akteuren in Konfliktkontexten lassen sich folgende Aufgabenfelder identifizieren:

- Veränderungen werden von Menschen geschaffen, daher müssen lokale zivile Akteure (change agents, GraswurzelDiplomaten) unterstützt werden, damit sie eigenständig gesellschaftlich-politische Veränderungsprozesse mit gewaltfreien Mitteln gestalten können. Konzeptionelle Ansätze gewaltfreier Transformationsprozesse müssen dafür nutzbar gemacht und weiterentwickelt werden. Die Beiträge externer und interner ziviler Akteure sollten daher genauer analysiert und weiterentwickelt werden.

- Lokale Friedenspotenziale müssen gestärkt werden, d.h. gesellschaftliche, kulturelle, politische und/oder ökonomische Faktoren mit transformativer Strahlkraft müssen bei Planungen von Programmen und Projekten identifiziert und anschließend gefördert werden. Hierfür gilt es systematisch friedensfördernde Instrumente zu entwickeln.

- „Un“zivile Akteure, die gewaltfreie Transformationsprozesse mit Gewalt unterlaufen, müssen identifiziert und für den angemessenen Umgang mit ihnen geeignete Instrumentarien entwickelt werden. Erste Untersuchungen dazu finden bereits statt.

- Konfliktsensibles Handeln (Do-No-Harm) aller externen Beiträge und damit eine Verhinderung der Verschärfung eines Konflikts ist ein wichtiger und essentieller Schritt.

- Krisenprävention, Konfliktbearbeitung und Friedensförderung müssen sich an Werten orientieren, die auch in Deutschland gelten, wie z.B. die internationalen Menschenrechte, völkerrechtliche Standards, Gewaltfreiheit und Gerechtigkeit.

\section{Empfehlungen über kurz- und mittelfristige Ziele}

Kurz- und mittelfristig sind folgende Kernempfehlungen umzusetzen, die im Folgenden näher konkretisiert und beispielhaft erläutert werden:

- Bestandsaufnahme und Lessons Learned: Rahmenbedingungen, Konzepte, Akteure, Instrumente, Ressourcen etc. dokumentieren, systematisieren und bisherige Ergebnisse und Erfolge sichtbar machen,

- Leitbild für zivile Friedensförderung und Krisenprävention entwickeln und daraus realistische Entwicklungsschritte definieren,

- Wirkungsmonitoring und Evaluationen: Erfahrungen evaluieren und dafür geeignete Instrumentarien entwickeln.

\subsection{Aktionsplan}

Die Erfahrungen mit der Umsetzung des Aktionsplans sollten sorgfältig analysiert, bisher Erreichtes identifiziert und die nächsten realisierbaren Ziele definiert werden.

\subsection{Ziviler Friedensdienst}

Die Ergebnisse der Evaluation des Zivilen Friedensdienstes sollten in einer Fachöffentlichkeit breiter diskutiert werden.

\subsection{Instrumente und Lessons Learned der zivilen Konfliktbearbeitung und Friedensförderung systematisieren und öffentlich machen}

Gewonnenes Wissen sollte nach der Rückkehr von Fachkräften nicht nur für interne Zwecke, sondern auch für die Fachöffentlichkeit zugänglich gemacht werden, damit deren Expertise der deutschen Politik, Gesellschaft und Forschung zugutekommen kann. Es könnten organisationsübergreifende Rückkehrer-Programme initiiert werden, um Erfahrungen der Friedensförderung und Konflikttransformation zu sammeln, zu systematisieren und um erworbene (Er-)Kenntnisse sichtbar zu machen. Ebenso sollten Lessons Learned systematisiert, zugänglich gemacht und diskutiert werden, um sie für die Planung neuer Programme und Projekte nutzen zu können. Dies könnte z.B. durch ressortübergreifende Fachveranstaltungen alle zwei Jahre und eine systematische Öffentlichkeitsarbeit geleistet werden, um die Erfolge ziviler Friedensmacher auf allen Ebenen sichtbar darzustellen.

\subsection{Zivile Expertise systematisch nutzen für Prävention und Interventionen}

Die Erfahrungen von Fachkräften in Krisenregionen können einen wichtigen Beitrag zur wirksamen Prävention leisten, indem Warnsignale drohender Gewalteskalationen frühzeitig erkannt und zur Kenntnis genommen werden. Gerade zivilgesellschaftliche Akteure auf lokaler Ebene haben ein gutes Gespür für Risiken und Potenziale der Gewalteskalation, aber auch lokal angepasste Ideen für wirksame und sinnvolle Interventionen. Ihre Einschätzungen könnten einen wichtigen Beitrag zum Frühwarnsystem der deutschen Politik leisten und innovative Ideen für zivile Interventionen liefern.

\subsection{Lernprozesse fördern}

Die Praxis der zivilen Konfliktbearbeitung sollte einem kollektiven Lernprozess gleichen. Sowohl die Politik als auch die Praxis betreten in diesem noch jungen Arbeitsfeld Neuland. Daher ist ein offener und ehrlicher Erfahrungsaustausch notwendig. Innerhalb der Institutionen, die in Deutschland Programme der zivilen Konfliktbearbeitung umsetzen, sollten ausreichende Ressourcen für Analyse- und Planungsprozesse lokaler Programme und Projekte sowie für systematische Evaluierungen und Lernprozesse bereitgestellt werden. Darüber hinaus sollte die kritische Reflektion bisheriger Programme und Erfahrungen ermutigt und honoriert und nicht „abgestraft“ werden. Nur durch eine kritische Betrachtung und die transparente Diskussion der eigenen Arbeit kann diese in Zukunft zielgerichteter und kontextspezifischer gestaltet werden. 
Diese Lernprozesse sollten sich nicht nur in der Praxis, sondern auch in der Politik etablieren. So sollten zusätzliche Ressourcen für Abstimmungsprozesse zwischen den Ressorts sowie für den Erfahrungsaustausch zwischen Politik, Forschung und Praxis bereitstehen. In diesem Zusammenhang müssen Dilemmata zwischen institutionellen und politischen Zielen, den Rahmenbedingungen sowie den Eigeninteressen der nationalen und lokalen Partner identifiziert und praktikable Lösungen zum Umgang mi ihnen gefunden werden.

\section{Empfehlungen über langfristige Ziele}

Internationales Engagement muss durch Capacity Building die Entwicklung einer aktiven und mündigen Zivilgesellschaft unterstützen. Langfristig angelegte Projekte sind daher notwendig, um Vertrauen aufzubauen und aufrechtzuerhalten sowie um Lernprozesse zu fördern und zu begleiten.

\subsection{Bundeshaushaltsordnung}

Deutschlands interne Vergabevorgaben und Verwaltungsmechanismen stehen der Begleitung langfristiger gesellschaftlicher friedensfördernder Transformationsprozesse im Weg. Die Bundeshaushaltsordnung sollte so gestaltet werden, dass sie die Bedürfnisse der Friedensförderung unterstützt: Förderung langfristiger Programme und Projekte (z.B. zehn bis 15 Jahre), institutionelle Förderung lokaler Partner (Infrastruktur, Personalkosten, laufende Kosten, Qualifizierungsprogramme) sowie Qualifizierungsangebote für zivilgesellschaftliche Akteure (Trainings, Informationsmaterialien, Studienreisen, Beratung, Coaching und Supervision). Dafür sollten Förderkriterien für zivile Programme und Projekte in Krisenregionen und Konfliktkontexten entwickelt werden, um die Effektivität und Nachhaltigkeit lokaler Akteure zu optimieren.

\subsection{Do-No-Harm und Friedensförderung für alle}

Für die Durchführung lokaler ziviler Projekte sollten vergleichbare Standards für alle externen Akteure gelten. Die Do-NoHarm-Kriterien haben sich für eine friedens- und konfliktsensible Projektarbeit der humanitären Hilfe, aber auch in der Entwicklungszusammenarbeit in Krisenkontexten bewährt und lassen sich auf andere Handlungsfelder übertragen. Die zentralen Erkenntnisse aus Fallstudien, die weltweit unter dem Stichwort Do-No-Harm bekannt sind, sind zum einen, dass jede Intervention im Kontext eines gewaltförmigen Konflikts Auswirkungen auf den Konflikt hat und zum anderen, dass jeder Konfliktkontext durch trennende Faktoren und Spannungen (Dividers) sowie verbindende Faktoren und lokale Potenziale für den Frieden (Connectors) bestimmt wird. Da jede Intervention in einer Wechselwirkung mit beiden Faktorengruppen steht, ist eine kontextspezifische und konfliktsensible Planung und Durchführung von Interventionen unabdingbar.

Weitere nützliche Instrumente sind die Ergebnisse des „Reflecting on Peace Practice“-Prozesses (RPP), der auf die Ver- besserung der Effektivität von Friedensarbeit abzielt. RPP hilft dabei Strategieentwicklung, Programmdesign, Monitoring und Evaluation zu stärken, um eine größere Wirkung zu erzielen. Auch dieses Instrument sollte daher flächendeckend bei der Planung und Auswertung von Programmen eingesetzt werden.

\subsection{Demokratieförderung/Good Governance}

Wahlen können für junge Demokratien oder Gesellschaften in Transformationsprozessen eine zentrale Herausforderung für die Demokratisierung und die Stabilität eines Landes darstellen. Viele Fälle haben gezeigt, dass vorschnelle Wahlen nicht ausreichen und oftmals zu neuer Gewalt in Post-Konflikt-Kontexten führen können. Daher müssen demokratische Wahlen in einen langfristigen demokratischen Lernprozess für alle Beteiligten eingebettet werden, damit sie friedensfördernd wirksam werden können. Dieser beinhaltet neben Bildung die Stärkung der Menschenrechte, der Minderheitenrechte sowie zivilgesellschaftlicher und politischer pro-demokratischer Kräfte und die Verbesserung der Leistung demokratischer Institutionen, um das Vertrauen der Bevölkerung in den Staat zu erhöhen.

\section{Kleiner Staat ganz groß:}

\author{
Finnlands Außen- und \\ Sicherheitspolitik 1995-2009
}

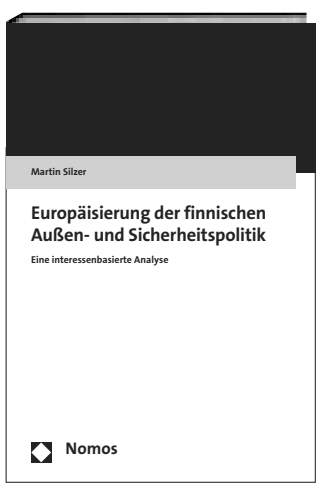

Europäisierung der finnischen Außen- und Sicherheitspolitik

Eine interessenbasierte Analyse

Von Martin Silzer

2014, 326 S., brosch., 59,-€

ISBN 978-3-8487-1424-7

www.nomos-shop.de/22739

Finnland ist mehr als Seen, Sisu, Sauna und Sibelius. Vielmehr war und ist das Land außerordentlich aktiv und erfolgreich beim Aufbau der europäischen Außenpolitik. Die Studie weist dies mithilfe eines Europäisierungsansatzes in Fallstudien aus der Militär- und Energiesicherheitspolitik nach und zeigt die Rückwirkungen auf das früher periphere Land auf. 